\title{
Marta Morazzoni e la ricerca della libertà
}

\section{Giuliana Sanguinetti Katz}

Marta Morazzoni è una scrittrice prolifica che nell'arco di dieci anni ha pubblicato ben quattro libri (La ragazza col turbante del 1986, L'invenzione della verità del 1988, Casa materna del 1992 e L'estuario del 1996). Fin dall'inizio i suoi libri sono stati accolti con grande entusiasmo dai critici. $L a$ ragazza col turbante è stata definita da Citati "un caso letterario" e Soldati vi ha visto un "romanzare alla Flaubert, che colma e placa". Il secondo e il terzo libro sono stati presentati al premio Campiello.

La sua caratteristica è di esprimersi attraverso immagini pregnanti di significato, dove descrizioni di personaggi, paesaggi e interni rappresentano particolari stati d'animo e dove le azioni più semplici acquistano un profondo significato psicologico. Come dice Citati: "Il suo dono maggiore è quello visivo. Essa vede una sensazione, a qualsiasi tipo appartenga; sa che in questa sensazione è concentrato tutto il futuro racconto, e . . trasforma la sensazione in narrazione".!

La Morazzoni dà inoltre alle sue immagini un particolare valore onirico, ponendole lontano da noi nella storia e nella geografia. Nel suo primo lavoro, La ragazza col turbante, su cui ci concentreremo in quest'articolo per esemplificare la sua arte, tutte le novelle, sono ambientate in tempi lontani: l'Austria di Mozart e di Salieri o dell'inizio di questo secolo, l'Olanda di Vermeer, la Spagna di Carlo V. Il fatto che si tratti di avvenimenti accaduti molto tempo fa, in paesi diversi, permette all'autrice di dare una struttura chiara e rassicurante ad eventi sconcertanti e talvolta orripilanti e di affrontare il tema della nevrosi e della ricerca di identità senza l'ansietà che viene da una completa identificazione del lettore con il personaggio.

In quest'opera l'autrice si serve della storia in modo diverso da molti scrittori di romanzi storici moderni, che sono mossi dal desiderio di vedere il passato come una preistoria, uno specchio, un'allegoria o una parodia del presente, o che mettono in questione la possibilità di conoscere sia il passato, sia il presente, facendo di tutto un gioco. ${ }^{2}$ La Morazzoni ispirata, come lei stessa dichiara, da "situazioni interiori, percezioni di stati d'animo", che hanno preso forma spontaneamente nella sua mente, si serve del filtro del passato per contenere l'emozione degli argomenti trattati. ${ }^{3}$ La storia con la sua chiarezza e 
razionalità funziona in questo caso da meccanismo di difesa della parte cosciente dell'io contro l'irrompere dell'inconscio. ${ }^{4} \mathrm{E}$ funziona anche come forza sublimatrice, perché la descrizione fascinosa di musicisti famosi, re, imperatori, nelle loro ville, palazzi e monasteri, sullo sfondo di paesaggi ora idillici ora pittoreschi, ci rappresenta l'importanza dei valori artistici nella nostra civiltà e la loro capacità di ridirigere i nostri impulsi distruttivi verso scopi più alti e di farci trovare il modo di superare le difficoltà che ci circondano.

Come nei sogni, il messaggio della narratrice, ci giunge dunque indirettamente, spostato nel tempo e nello spazio e presentato con una forma classica che ne nega il contenuto nevrotico. Come osserva acutamente la Rigobello: "Il passato è osservato come una galleria di quadri. . scrutati con scrupolosa attenzione e con finissima sensibilità, contemplati con adesione sentimentale costantemente frenata da lucida razionalità, con realismo e contenuto lirismo" (16). Classico è anche il linguaggio della Morazzoni con i suoi periodi "ampi e riposati", il suo uso della dittologia, dell'iterazione e delle sinonimie (Rigobello 18) e il suo senso del ritmo legato al suo amore per la musica. ${ }^{5}$

Altre caratteristiche che rendono i lavori della Morazzoni simili ai sogni sono il suo costante uso di omissioni (personaggi e scene importanti che vengono omesse dal testo, e lasciate all'immaginazione del lettore) e l'allusività delle scene descritte, soprattutto nel caso delle descrizioni di ambienti e di paesaggi. Le omissioni danno un senso di mistero inquietante al testo, che turba e coinvolge il lettore, e le descrizioni condensate e allusive hanno il valore di ricordi, che assommano in sè complessi stati psicologici ${ }^{6}$ Tipicamente psicologico è anche l'uso del tempo, con narrazioni molto lunghe e lente di brevi periodi di tempo particolarmente significativi e rapidi accenni a lunghi periodi della storia, trattati come meno importanti.

Se passiamo ora alla psicologia dei personaggi, vediamo che vivono in ambienti rigidamente controllati da regole di vita che, da una parte, danno loro un punto chiaro di riferimento, ma dall'altra ne soffocano i sentimenti e i bisogni istintuali. Eccezion fatta per il crudele e ipocrita Da Ponte nella storia "La dignità del signor $\mathrm{Da}$ Ponte", i protagonisti dei racconti sono persone colte e oneste, dotate di un forte senso morale, che proprio da tale senso morale vengono inibite e rischiano di rimanerne sacrificate. Queste persone, tuttavia, non sono completamente chiuse nei loro rituali e, talvolta, messe davanti a momenti di crisi, malattie gravi, rivolgimenti famigliari, trovano la forza di ribellarsi alla loro routine e di forgiarsi o di accettare un nuovo modo di vivere.

La prima opera della Morazzoni mostra l'abilità stilistica dell'autrice e così pure il suo fine intuito psicologico nell'esplorare gli stati d'animo di vari personaggi, colti tutti in momenti cruciali della loro vita. Si tratta di una raccolta di cinque racconti, intitolata La ragazza col turbante, dal titolo del racconto centrale. Gli argomenti trattati sono vari: la morte di un musicista, molto simile a Mozart, che ritrova negli ultimi istanti della sua vita la gioia dell'infanzia; la 
discesa del poeta Lorenzo Da Ponte in una Vienna infernale, e la serie di umiliazioni che subisce, che lo portano a commettere un omicidio; il viaggio di un mercante d'arte per vendere un suo amato quadro di Vermeer, proprio prima che sua moglie partorisca un figlio; la decisione dell'idalgo Quisada di ribellarsi alla tirannia capricciosa di Carlo V e infine la "rinascita" di un industriale viennese, rimasto paralizzato da un infarto, che nelle mani di un fedele servitore e di un sollecito dottore impara a gustare di nuovo i piaceri semplici della vita.

Mentre i primi due racconti ci presentano due situazioni estreme di paradiso e inferno, il racconto centrale ci mostra l'importanza di rinunciare a certi sogni e piaceri dell'adolescenza per affrontare le responsabilità della maturità. Gli ultimi due racconti, infine, ci conducono fuori del labirinto delle convenzioni sociali e ci indicano la via verso un modo di vivere più sano e in armonia con la natura. La forza e l'evidenza di questi racconti viene anche dal fatto che la Morazzoni si rifa a schemi mitici e fiabeschi che ci riportano a ben note situazioni: la visita alla casa misteriosa, l'errare per una città ostile con movimenti concentrici che riportano il viandante sempre allo stesso posto, il viaggio difficoltoso per mare alla ricerca di una terra sconosciuta, il percorso attraverso la foresta, il volo in alto al di là della prigione della casa, grazie alle ali della fantasia.

In particolare il mito del labirinto (mito suggerito anche dal nome di Arianna, dato a uno dei personaggi nel racconto centrale della raccolta) sembra assommare in sè il significato principale dell'opera. Tutti i racconti trattano di viaggi difficoltosi e penosi che il protagonista deve percorrere, affrontando la morte, per giungere al centro del labirinto della propria psiche e rinascere a nuova vita (Santarcangeli 355-361; Eliade 67, 70, 84). Talvolta i protagonisti rimangono chiusi nel labirinto, o perchè con un atto di eccessiva brutalità distruggono ciò che di buono poteva ancora esserci nella loro anima (Da Ponte che uccide il mendicante), o perchè sono prigionieri di ricordi e affetti della loro adolescenza (il mercante d'arte, che ha lasciato il suo cuore con il quadro di Vermeer). Altre volte, invece, riescono a superare la morte, l'infermità e la soggezione a persone crudeli e dominatrici, andando indietro nel tempo e rivivendo in maniera positiva i momenti lieti della propria vita (il compositore Karl che ride felice come un bambino nell'aprire l'ultima porta della villa; Quisada che fa all'amore con la zingara incontrata nella foresta; Karl Kolner che ripensa al moscato bevuto da ragazzo con la madre e il fratello, nel corso di una vacanza). ${ }^{7}$

Il primo racconto, La porta bianca, ha luogo nel settecento e descrive l'ultima giornata di un compositore, di nome Karl, che, ospite di misteriosi signori, cerca invano di comporre un Requiem. Inquietante è la lussuosa casina di caccia in cui è ospitato il maestro, con le sue finestre "troppo grandi in proporzione alla casa, così che questa sembrava un cristallo senza sostegno di mura, spalancata alla curiosità di tutti” (10). Angoscioso è poi il giardino, dove 
tante gradazioni di verde "si confondevano nella loro uniforme varietà, ma nessun colore interrompeva il chiaro e lo scuro delle piante e dell'erba, che si esaurivano solo nel grigio massiccio della villa emergente nello sfondo" (14). La casa e il giardino in realtà riflettono la condizione fragile e ansiosa del protagonista, che si sente "astiosamente minacciato da un cupo fermento nemico" e a cui talvolta pare di "essere chiuso in un gorgo di malesseri indistinti" (9).

Dopo una notte affannosa, passata in poltrona, Karl viene visitato la mattina seguente dalla moglie Costanza, frivola, capricciosa e infantile. Costanza nota il pallore, il precoce invecchiamento del marito, che era prima suo compagno di giochi. Quando questi le vieta di avvicinarsi alla villa dei misteriosi padroni per non disturbarli, lei irritata lo lascia e se ne torna a Vienna. La partenza di Costanza, con le sue allegre risate e la sua voglia di vivere, rappresenta la fuga delle forze vitali dal corpo e dalla mente di Karl. Lei è inoltre la moglie bambina che non può e non vuole dare al marito quelle cure materne di cui lui ha bisogno.

Venuta la notte e rimasto solo, il compositore si addormenta e "in una regione dove pensiero e realtà si uniscono" immagina che il momento sia finalmente venuto di incontrare i padroni della villa (24). Divenuto piccolo e debole come un bambino e vestito a nuovo con scarpini di vernice e giacca di raso, viene scortato da camerieri indifferenti o addirittura ostili attraverso porte difficili da aprire, lungo il viale erboso che conduce alla villa, su per la gradinata su cui si innesta "la solenne vertigine del colonnato" (25). Una volta penetrato nella villa si trova prima in un "androne scuro, umido e pesante" (26), dove deve aspettare lungamente al buio e al freddo e piange disperato, poi apre una porta bianca laccata ed entra in una stanza alta e luminosa, con una luce bianca "riflessa in un'infinita fuga di specchi" e dei tendaggi color del miele (28). Al fondo della sala lo attende "una semplice porta di legno bianco e compatto" (la porta bianca del titolo), al di là della quale il compositore vede qualcosa che lo fa scoppiare in una risata cristallina che sembra "percorrere a ritroso la sala, l'androne buio, la scalinata oltre le colonne e frangersi contro le sbarre del cancello, in fondo al giardino" (29).

In questo caso, nel suo viaggio verso la morte, Karl ripercorre all'indietro le tappe della sua vita. L'androne di pietra della villa, le maniglie dure da aprirsi, i camerieri ostili alludono probabilmente alla giovinezza e all'infanzia difficili del protagonista: "gli anni della prima gioventù . . . angustiati dall'angoscia di sentirsi rinchiuso in una dimensione senza futuro" (10-11) e il periodo dell'infanzia "quando, bambino, aveva girato mezza Europa per esibire come un mostro da fiera il suo talento prodigioso" (22). Tempi infelici, condensati nel ricordo della carogna di un cane schiacciato da una carrozza e abbandonato al margine della strada (11). In contrasto con gli ambienti precedenti, la stanza chiara e luminosa, tutta specchi, e tende color miele, che conduce alla misteriosa porticina bianca, si riferisce probabilmente ai primi mesi di età del bambino, 
quando è allattato dalla mamma e dipende completamente da lei. Le tende color miele sono un possibile riferimento al latte, visto l'accoppiamento consueto dei due sostantivi nell'espressione "latte e miele". Gli specchi sembrano alludere al fatto che il bambino in questo periodo dello sviluppo si rispecchia nel mondo circostante, cioè vede quello che lo circonda solo in termini di oggetti di desiderio o di timore. ${ }^{8}$

L'autrice non dice al lettore incuriosito che cosa giaccia dietro la porta bianca del titolo e rispetta il mistero della morte del protagonista. Tuttavia la struttura allusiva del racconto spinge il lettore a ricostruire l'ultima scena che si apre alla vista di Karl. In questo viaggio a ritroso lungo la vita del protagonista, la piccola porta, con la maniglia minuta e leggera, sembra condurre al ventre materno: ciò spiegherebbe la lunga risata cristallina di Karl, finalmente libero da ogni angoscia e preoccupazione, senza alcun dovere o dolore. In questo racconto, dunque, il peso di una vita costretta dalle convenzioni e dalle necessità economiche, si risolve in una fuga nel tempo verso una morte che è concepita come un atto di liberazione e un possibile inizio di una condizione serena e felice. Il viaggio all'indietro attraverso il labirinto delle sofferenze della vita porta alla fine a una rinascita nel grembo materno. ${ }^{9}$

Nel racconto seguente "La dignità del signor da Ponte" vediamo la società della Vienna di Mozart rappresentata come "un nido di vipere" (33), un vero inferno, al centro della quale c'è il diabolico Salieri, pieno di "ostilità cattiva" e di "malvagio gusto di ferire" (56). Questi è descritto come un essere stupido, vano e crudele: acconsente di mala grazia a servirsi di Da Ponte come librettista per una sua opera e fa lavorare l'abate senza tregua, facendogli cambiare ogni scena del suo libretto all'ultimo momento. Infine, quando l'opera è un fiasco, tratta Da Ponte crudelmente, attribuendo a lui tutta la colpa dell'insuccesso e scacciandolo in malo modo.

Da Ponte, da parte sua, è un essere suadente, rapace e infido, che nasconde i suoi vizi "dietro la melliflua ipocrisia di un volto dabbene" (33) e che si compiace di raggirare il prossimo. Spinto dal bisogno economico e dall'ambizione, si mette al completo servizio di Salieri, cerca in ogni modo di conquistarne la stima e passa i giorni nei caffè e nei salotti di Vienna, nel vano tentativo di farsi una posizione. Le visite penose e umilianti di Da Ponte a Salieri sono sottolineate dalla presenza di uno straccione vicino alla casa di Salieri, "una indefinita figura scura, non molto alta e tanto celata da un ampio mantello marrone, da non potersi riconoscere se fosse uomo o donna" (38). Da Ponte la prima volta che lo incontra teme che si tratti di un assassino, non vuole provocarne l'ira e perciò non cerca di evitarlo attraversando la strada. Con ribrezzo e terrore sente il momentaneo contatto con lo straccione che gli preme contro il fianco "qualcosa di molle" (38). In realtà si tratta di un povero mendicante, che rimane immobile al freddo e alla pioggia nella speranza che qualcuno si prenda cura di lui. 
Quando Salieri tratta con disprezzo Da Ponte e non gli suggerisce nessun argomento per un libretto, questi è umiliato e pieno di "un astio velenoso e cattivo" (53). Incontrando per la seconda volta il mendicante vicino alla casa di Salieri, l'abate gli augura sottovoce con preghiera diabolica "di patire la fame in questo mondo e nell'altro" (53) se spera di vivere della carità del signor Salieri. Infine, quando è completamente umiliato da Salieri durante il loro ultimo incontro, Da Ponte sfoga la sua ira terribile sul povero mendicante che si trova vicino al portone, lo colpisce violentemente al viso e al petto e lo lascia a terra mezzo morto, "un fagotto pulsante e insanguinato" (58). Poi Da Ponte seppellisce quest'episodio nel suo inconscio e solo di tanto in tanto il ricordo riaffiora come "una puntura di vespa che lo trafiggeva talora, a tradimento, e per la durata di un attimo gli alterava la fisionomia del volto" (59).

Da Ponte, dunque, proietta le sue paure, rabbie e miserie sul mendicante: lo vede a volta a volta come un pericoloso aggressore e come un povero idiota. Man mano che è sempre più umiliato da Salieri, Da Ponte si rispecchia nel mendicante stracciato e bisognoso. Egli vede se stesso come uno straccione qualunque, costretto a mendicare un sorriso o una buona parola del maestro. Alla fine, questo sadico gioco di umiliazioni finisce nel delitto più brutale, dove è il debole e l'innocente a pagare il fio della cattiveria altrui. La dignità dell'uomo si riduce a mera apparenza, una maschera dettata dalle convenzioni sociali, dietro a cui si nasconde il volto dell'assassino. In questo caso Da Ponte, nel suo aggirarsi per il centro di Vienna, in direzione di casa Salieri, cade sempre più in basso, finché alla fine rimane chiuso nel diabolico labirinto della propria brutalità. ${ }^{10}$

Mentre i due primi racconti esaminano il rapporto tra l'artista e una società crudele, opprimente e sfruttatrice, il terzo racconto, che dà il nome alla raccolta, esamina l'individuo in termini non di problemi sociali, ma di problemi personali causati da conflitti non superati nel proprio sviluppo. La storia del mercante d'arte olandese, che abbandona la moglie incinta per andare a vendere un suo amatissimo quadro di Vermeer "La ragazza col turbante" a un nobile danese, rappresenta una variante di una particolare situazione psicologica. L'adulto che non ha superato il suo complesso di Edipo, messo di fronte alla sessualità della moglie nel corso della gravidanza, non la tollera, perchè gli ricorda il proprio attaccamento incestuoso alla madre e perchè lo minaccia con la presenza di un futuro rivale. Egli si rifugia allora in fantasie della madre, vista come creatura pura e adolescente, tutta dedita a lui, e talvolta inizia un rapporto con un'altra donna, che possa offrirgli quest'alternativa. ${ }^{11}$

Il mercante Bernhard Van Rijk, solo nel suo letto matrimoniale, poiché la moglie incinta si è ritirata in una camera più appartata, assistita da una serva, ha sostituito la presenza della moglie con il quadro della affascinante ragazza col turbante. Bernhard tiene il prezioso quadro nella camera da letto, lo guarda ogni sera prima di coricarsi e lo "gratifica(va) di una gelosa, paterna tenerezza" 
con comprensibile astio della moglie (68). Lui si allontana ulteriormente dalla moglie, benché questa sia visibilmente angosciata dai pericoli della gravi-danza, e si imbarca in un viaggio in Danimarca, destinato a durare due mesi, nel corso del quale venderà il suo amato quadro a un nobile danese.

In Danimarca incontra l'anziano sire di Herfolge, il possibile acquirente del quadro, che vive appartato da tutti nelle sue terre con la sua giovane figlia Arianna. La vita regolata e pacifica del nobile danese, cosi lontana dalla vita affannosa del mercante, e la spontaneità e innocenza di sua figlia Arianna, rappresentano per Bernhard un paradiso terrestre dove amerebbe fermarsi. Arianna e suo padre lo invitano a soggiornare più a lungo, così come Nausica $\mathrm{e}$ Alcinoo invitano Odisseo a rimanere con loro nell'isola dei Feaci. Arianna mostra una chiara simpatia per il mercante e il signore di Herfolge lo tratta affettuosamente come un figlio, ma Bernhard, timoroso delle conseguenze di tale incartevole ozio, decide di partire il più presto possibile. In realtà il rapporto di Bernhard con il suo quadro si rispecchia nello stretto rapporto del sire di Herfolge con sua figlia Arianna. L'attrazione del mercante per Arianna rappresenta un sentimento incestuoso e quindi proibito. Lui deve allontanasi dalla fanciulla, cosí come deve lasciare dietro di sè il quadro tanto amato, per poter affrontare degnamente i suoi doveri di pater familias.

Tuttavia, dopo il suo ritorno in patria, la vita del mercante sembra perdere ogni interesse e viene narrata in meno di una pagina. Non si sente più parlare della moglie e si apprende invece che il mercante ha avuto un unico figlio, Jan, bello e intelligente, che segue in tutto e per tutto le orme del padre e continua a vivere nella casa paterna. Jan, come Bernhard, è affascinato dal commercio, che segue puntigliosamente, e solo dietro richiesta del padre morente, si decide a prendere moglie. Alla fine dei suoi quarant'anni gli giunge una lettera di Arianna di Herfolge che, costretta a separarsi dalla sua casa e dai suoi possedimenti, vuole che il dipinto tanto amato da Bernhard, dal sire di Herfolge e infine da lei, finisca nelle mani di Jan, in nome dell'affetto che lo lega alla memoria di suo padre. Dopo aver ricevuto questa lettera, Jan si affretta a partire per il suo primo viaggio in Danimarca, ripercorrendo la rotta del mare del Nord, seguita da suo padre proprio prima che lui nascesse.

La vita di Bernhard sembra dunque essersi fermata al suo viaggio incantato in Danimarca e al suo incontro con Arianna e con il signore di Herfolge. Allo stesso modo il figlio Jan, che è come uno specchio del padre, sembra non avere una sua vita sentimentale e sembra svegliarsi solo alla lettera di Arianna che lo richiama al passato e che gli consegnerà il prezioso quadro, prediletto dal padre. Il riapparire di un'Arianna ormai invecchiata e del quadro della ragazza col turbante ha il significato di una rivelazione amorosa. Il quadro che Arianna darà a Jan è l'equivalente di un ritratto di Arianna com'era al tempo in cui Bernhard l'ha incontrata. Jan capirà allora l'amore segreto che ha vincolato il padre per tutta la vita e dovrà sciogliere l'incantesimo che ha tenuto legato 
anche lui nell'ombra del padre. Arianna, come la principessa del mito di Teseo, aiuterà forse Jan a uscire dal labirinto di antichi avvenimenti, rimasti sepolti nell'inconscio.

Come negli altri racconti del libro, anche qui le descrizioni di interni e di paesaggi sono molto significative. In particolare il viaggio di Bernhard in Danimarca ben rende il senso di sogno e di ritorno al passato che domina l'animo del mercante. La nave di Bernhard scompare all'orizzonte come un fantasma inesistente e il mercante vede la sua città "riassorbita nell'uniforme grigiore della linea costiera" (75). Man mano che lascia la costa e affronta il mare aperto, Bernhard si perde "in lunghe divagazioni senza senso" e si abbandona a "un singolare stato d'animo" che "si opponeva all'oggettività dei fatti e li sovvertiva" (80). Si sente incerto e sospeso nella condizione provvisoria del viaggio e non ne vede la fine: una strana noia lo invade. Una sera, quando il viaggio sta per terminare, va a dormire ed è sopraffatto da "un avvicendarsi di fantasie che non avevano nulla a che fare con la gravità di un uomo maturo" (82) e sente dentro di sè la voce del capitano che gli chiede che età ha. Infine, al suo arrivo nel palazzo del signore di Herfolge, si addormenta "di un sonno profondo e faticoso", spossato dal viaggio.

Si ha l'impressione che Bernhard compia un viaggio nel tempo e nello spazio, simile a quello di Silvestro in Conversazione in Sicilia di Vittorini. Ma mentre Silvestro riassapora le delizie dell'infanzia e della giovinezza vicino a sua madre Concezione, pur rimanendo ben conscio del presente, Bernhard perde il contatto con la sua vita presente in Olanda, si sente sradicato e disorientato, affetto da un'inspiegabile ansia. Anche il paesaggio danese all'inizio gli sembra ostile e poco attraente: la "selvatica irrequietezza della terra collinare" (96) e i "colori intensi e brillanti" dei campi coltivati che a Bernhard sembrano "eccessivi e carichi di un'energia prepotente, aggressiva" (85) ben rappresentano lo stato d'animo del mercante.

Bernhard regredisce nel tempo e rivive sentimenti di libidine e di aggressione a lungo repressi. Egli, da una parte si sente rassicurato dalle maniere affabili e signorili del signore di Herfolge e di sua figlia e dal loro modo di vivere così tranquillo e ben regolato, dall'altra si sente minacciato dai modi diretti di Arianna e dall'invito che lei gli fa a rimanere con loro al castello. La disponibilità del sire di Herfolge e di sua figlia nei riguardi di Bernhard potrebbe rappresentare una fantasia edipica del figlio, che vorrebbe che il padre gli offrisse il suo posto vicino alla madre e che la madre fosse molto giovane e inesperta, cosí che il figlio potesse proteggerla e guidarla, rovesciando i ruoli reali. Tale fantasia nasconde l'originaria rivalità del figlio verso il padre e la sua conseguente ansietà di castrazione, espressa nel racconto dalle descrizioni ansiose del viaggio in mare e delle colline e della campagna danese.

La condizione psicologica del protagonista e di suo figlio è sottolineata dalla struttura del racconto dove l'iniziale descrizione dettagliata di Jan, il 
figlio di Bernhard, e della casa dei genitori in cui vive, serve da introduzione al viaggio in Danimarca di Bernhard, viaggio che come abbiamo visto, sembra quasi concludere la vita del mercante. Solo alla fine del racconto si ritorna brevemente alla storia di Jan, e al viaggio che questi intraprende sulle orme del padre alla ricerca del famoso ritratto. Cioè il fatto che Jan sia solo un'ombra del padre è reso appunto dal modo sommario in cui l'autrice si sbriga di lui, lasciandoci aperta la conclusione della storia.

I due seguenti racconti ci riportano di nuovo al tema dei primi due. Anche qui i protagonisti sono vittime di un ambiente soffocante e di una ferrea disciplina, ma, diversamente dai personaggi iniziali, riescono a opporsi alle convenzioni della società e ad affermare l'importanza di un nuovo modo di vivere. Anche in questi racconti le descrizioni di ambienti e paesaggi giocano un ruolo cruciale e rappresentano i vari stati d'animo dei personaggi principali.

Nel quarto racconto, "L'ultimo incarico", Don Luis Quisada, costretto a sacrificare la sua vita famigliare per fare da maggiordomo a Carlo V, si ribella al dispotico sovrano e va a incontrare una zingara che lo aspetta vicino al convento dove risiede l'imperatore. La vita di sforzi e sacrifici quotidiani che Don Luis Quisada deve fare per accontentare i capricci del sovrano, contrasta con la vita libera e istintiva della zingara, che è una creatura delle selve, lontana dalla disciplina e dai valori della società. Alla fine Don Luis riconosce l'importanza di un modo di vivere più naturale, si concede un incontro amoroso con la donna e, morto l'imperatore, brucia il manoscritto delle memorie di Carlo, che questi lo ha obbligato a leggere per lunghi giorni.

La servitù di don Quisada, che quasi muore di un'infezione polmonare nello sforzo di compiere il suo duro lavoro, è ben resa dagli ambienti delle miserabili locande dove è costretto a sostare, o dal paesaggio montuoso dell'Estremadura, che deve percorrere al servizio dell'imperatore. D'altra parte il bosco che Quisada deve attraversare ogni giorno, per andare a Yuste a far provviste di cibo, rappresenta il temperamento "irruente e sincero" dell'idalgo, che mal si adatta alla vita di corte (109).

Una mattina che Quisada attraversa il bosco, vede emergere dalla nebbia e dal fogliame una vagabonda che si muove con passo felino, quasi "una di quelle suggestioni vane che dalla nebbia aggrediscono la fantasia" (117). La donna non è piú giovane, ha i capelli sporchi e lunghi, dorme per terra, come un animale, e parla di Quisada come di "un servo del re che sta chiuso a Yuste" (118). Tra la donna e l'idalgo si stabilisce un'intesa, nata dai loro incontri quotidiani nel bosco. Quando l'idalgo, nella sua camminata mattutina a prendere rifornimenti, si trascina malatissimo per la strada del bosco, con la botticella del latte sulla schiena, è la vagabonda che interviene ad aiutarlo e a portare la botticella per lui.

Dopo la malattia quasi mortale che ha avuto, Quisada si ribella alla volontà dell'imperatore, raggiunge nel bosco la zingara salvatrice, fa l'amore con lei, e 
prova poi "il benessere del sole e del silenzio" mentre "nelle vene, fatte larghe e placide come gi argini vasti del Tago, il sangue scorre(va) tranquillo e appagato" (139). La ribellione a Carlo e l'unione con la vagabonda sembrano aver dato a Quisada una gran pace, sicurezza di sè e capacità di apprezzare la natura. Infatti, come per miracolo, da quel momento Carlo V è colto dalla febbre terzana e si avvia alla fine, che segnerà la liberazione di Quisada e del seguito dell'imperatore. La prova del distacco definitivo di Quisada dalla corte e del suo odio di un certo tipo di vita ipocrita e prepotente, è l'atto simbolico con cui, ritornato a casa, brucia il manoscritto delle memorie di Carlo V nel caminetto della cucina.

Nel quinto raconto, "L'ordine della casa", che si svolge a Vienna all'inizio del novecento, il signor Karl Kolner, colpito da paralisi, è costretto a giacere immobile in casa senza potersi muovere e senza parlare, mezzo sordo e costretto a comunicare solo con gli occhi. La vita del signor Kolner, prima puntigliosa, regolata e convenzionale, acquista un nuovo signicato ora che lui deve imparare a comunicare in modo nuovo con il suo medico e che ogni movimento del suo corpo è una vittoria sulla malattia. I nuovi rapporti che il malato ha con il suo medico curante e con il cameriere che lo assistono devotamente rappresentano una vera rinascita.

Questi segni di vita nel signor Kolner sono contrastati, tuttavia, dalla moglie di lui, una donna dura, metodica e crudele, che pensa solo a mantenere le apparenze e si augura che il marito, cosí deformato e menomato com'è, muoia il più presto possibile. Quando l'energico medico Ober vuole persuadere la signora Kolner a lasciare che il marito esca in poltrona a rotelle a prendere un po' d'aria o addirittura vada in montagna, costei inorridisce all'idea che qualcuno possa vederlo in quello stato e manda via il povero medico. Tuttavia, anche se la signora Kolner nega al malato le cure di cui avrebbe bisogno, questi, tramite la forza della fantasia e del ricordo, evade dalla prigione del suo corpo paralizzato e della casa in cui è rinchiuso. Inoltre, con la sua stessa presenza, mette in crisi i valori "estetici" di cui sua moglie si fa una ragione di vita.

L'importanza psicologica dei paesaggi e degli ambienti è particolarmente evidente in questo racconto. Per la signora Kolner, che passeggia a Schonbrunn, il terrore che suo marito appaia in pubblico è tale che lei ha un'allucinazione e immagina con raccapriccio di vedere "il grottesco veicolo [del marito] nel viale bianco di polvere" che conduce alla reggia (170). Corre allora a pregare la sorella di non chiedere un permesso speciale alla cancelleria affinché il marito possa andare a Schonbrunn, e chiude definitivamente in casa il signor Kolner. In questo caso lo schermo giallo della reggia di Schonbrunn, con la sua cancellata, l'androne, il porticato, i giardini, e "alla sommità della linea precisa dei cipressi, alta sulla collina . . . la sagoma nera in controluce della Gloriette" (170-171) ben rappresentano il senso di decoro, il rispetto delle convenzioni, l'eleganza che formano lo stile di vita della signora. 
D'altra parte le vedute di Vienna alla fine del secolo, il tram a cavalli che congiunge la periferia boscosa al centro, il Caffè Centrale con i suoi tavolini affollati, la messa a Santo Stefano il sabato mattina alle undici, la "luminosità calda e misteriosa della cattedrale" (160) la notte di Natale, la magnolia fiorita nel giardino di villa Kolner, sono tutte pensate con struggente nostalgia dal signor Kolner, che giace paralizzato nella sua casa. Quando sente la voce del suo medico che vorrebbe portarlo in montagna, lui ricorda "un paese della Svizzera italiana, dove da ragazzo aveva una volta, con la madre e il fratello maggiore, passato una vacanza" (172) e immagina di tornare lì a bere del moscato italiano per il suo compleanno. E sono appunto i suoi ricordi del passato e la sua capacità di gustare i minimi piaceri del presente che rappresentano per lui una fuga dalla prigione del suo corpo e soprattutto dall'opprimente presenza della moglie. In questo caso la fantasia e l'immaginazione aiutano il signor Kolner a librarsi al di sopra del labirinto in cui è rimasto rinchiuso e a evadere in un mondo più sereno.

In conclusione i valori umani fondamentali e le necessità istintuali dell'individuo vengono rappresentati in tutti $\mathrm{i}$ racconti, in contrasto con la durezza repressiva della società e la crudeità dell'uomo verso il suo prossimo. Da una parte il fascino della storia, dei personaggi famosi e dei luoghi lontani, i richiami fiabeschi e mitologici servono a distanziare gli avvenimenti, ad attutirne l'impatto e a dare alle vicende narrate un valore universale. Dall'altra la sequenza di immagini con forte valore onirico contribuisce a coinvolgere il lettore e a renderlo fortemente partecipe dello stato d'animo dei protagonisti.

Ogni racconto, pur nella minuzia e precisione dei suoi dettagli realistici, si presenta con un suo significato simbolico nascosto, che impone al lettore una lettura attenta e concentrata. Le pause, le omissioni di situazioni chiave (per esempio quello che il compositore vede dietro la porta bianca nel primo racconto), lo sparire di personaggi importanti (per esempio la moglie incinta nel terzo racconto), costringono il lettore a lavorare di fantasia e a riempire i vuoti con una possibile spiegazione. In questo modo il lettore è attirato per forza nel mondo dell' autrice e finisce per partecipare attivamente alla creazione del racconto che riverbera nella sua mente con una molteplicità di echi e di allusioni.

\section{University of Toronto, Erindale College}

\section{NOTE}

1 Si veda a questo proposito anche l'articolo della Rigobello, che esamina lo stile della Morazzoni e lo paragona alla musica e alla pittura, dando particolare rilievo alle capacità descrittive e evocative della scrittrice.

2 Si vedano l'articolo di Rosa "Di storia" e l'intervista che la stessa autrice fa a Remo Ceserani in "Cinque domande" (24-36). 
3 Vedi le dichiarazioni della Morazzoni sul suo modo di comporre nell' intervista da lei concessa a Giordano.

4 Anche la Mazza mette in evidenza il contrasto tra l'accurata ambientazione storica e gli elementi puramente fantastici e nevrotici degli argomenti trattati. Tuttavia la Mazza vede "la malattia, la nevrosi, la solitudine" non come fenomeni comuni di tutti i tempi ma come interessi tipici della nostra epoca. Lei ritiene, perciò, che la Morazzoni appartenga alla cultura del neo-decadentismo del ' 900 , cioè proietti nel passato situazioni di angoscia e di nevrosi tipicamente moderne, che la affascinano (807).

5 Si veda la dichiarazione della Morazzoni a Giordano a proposito della scrittura: "Mi capita spesso di associarla alla stessa tessitura di un discorso musicale, di doverne cercare in qualche modo la medesima trama armonica, il senso del ritmo, che è essenziale anche e soprattutto in una pagina di prosa". Tale affermazione della Morazzoni è citata e ampiamente commentata dalla Rigobello nel suo articolo.

6 A proposito dell'uso che la Morazzoni fa della storia, Citati parla della sua grande capacità evocativa che non ha bisogno di accumulare dettagli e della sua abilità di omettere e falsificare avvenimenti, creando così una "cristallizzazione storica, mentre il velo del tempo scende e allontana le immagini da noi".

7 L'identità del nome del primo e dell'ultimo protagonista sottolinea la somiglianza psicologica dei due personaggi e la loro capacità di evadere dalla prigione della loro vita.

8 Per una descrizione del primo sviluppo del bambino si veda Fenichel 33-41.

9 Per un'interpretazione del viaggio nel labirinto come penetrazione dell'alvo materno, si veda Santarcangeli 65 .

10 Per una particolare versione del labirinto come l'inferno con il diavolo al centro, si veda Santarcangeli 248. Il più famoso esempio di quest'interpretazione del labirinto la troviamo nella Divina Commedia.

11 Per i sentimenti di rivalità che il bambino prova normalmente verso i fratellini e il suo timore che la mamma rimanga incinta e gli procuri nuovi rivali si veda Freud, "Introduzione"; si vedano anche le fantasie nevrotiche del piccolo Hans in Freud, "Analisi", e l'analisi del sogno di Freud riportata da Jones 38-39. Per quel che riguarda la divisione tra la donna sessuale e pericolosa (la madre sessuale) e la donna pura e idealizzata (la madre che nutre e protegge il figlio) si veda l'articolo di Freud, "Sulla piú comune". L'uomo che non ha superato il complesso di Edipo spesso separa i due lati della madre (e della donna in genere) per difendersi dai suoi sentimenti incestuosi verso la figura materna. Di solito può amare solo donne che vede come madri amorose e pure, mentre disprezza e degrada le donne viste come esseri sessuali. Bernhard vede la gravidanza di Miriam come chiaro segno di sessualità e quindi fugge da lei e vagheggia l'immagine di fanciulle caste e idealizzate.

\section{OPERE CITATE}

Citati, Pietro. "Morazzoni: la storia immobile". Corriere della Sera 14 maggio 1986.

Eliade, Mircea. Images et Symboles - Essais sur le symbolisme magico-réligieux. Paris: Gallimard, 1952.

Fenichel, Otto. The Psychoanalytic Theory of Neurosis. New York: W. W. Norton \& Company, 1972.

Freud, Sigmund. "Analisi della fobia di un bambino di cinque anni". Opere 5.555-88.

"Introduzione alla psicoanalisi". Opere 8.489-95.

Opere. Ed. Cesare Musatti. 12 voll. Torino: Boringhieri, 1967-80.

"Sulla più comune degradazione della vita amorosa". Opere 6.421-32.

Giordano, Luigi. "Amori e odi in bella scrittura". Il Maltino 7 agosto 1986.

Jones, Ernest. The Life and Work of Sigmund Freud. Ed. L. Trilling and S. Marcus. Harmondsworth:

Penguin Books, 1964.

Mazza, Antonia. "La ragazza col turbante”. Lellure 41.431 (novembre 1986): 807-8. 
Morazzoni, Marta. La ragazza col turbante. Milano: Longanesi, 1986.

Rigobello, Giuliana. "Marta Morazzoni ovvero la scrittura come pittura e come musica". Coscienza 4 (1993): 16-19.

Rosa, Giovanna. "Di storia in storia". Spinazzola 9-23.

"Cinque domande sul ritorno al passato". Spinazzola 24-96.

Santarcangeli, Paolo. Il libro dei labirinti. Firenze: Vallecchi, 1967.

Soldati Mario. "Il bello è che non succede mai niente". Corriere della Sera 14 maggio 1986.

Spinazzola, Vittorio, ed. Tirature '91. Torino: Einaudi, 1991. 